\title{
Radiofrequency ablation for the treatment of non-small cell lung cancer in marginal surgical candidates
}

\author{
Hiran C. Fernando, MD \\ Alberto De Hoyos, MD \\ Rodney J. Landreneau, MD \\ Sebastian Gilbert, MD ${ }^{\mathrm{a}}$ \\ William E. Gooding, MS ${ }^{b}$ \\ Percival 0. Buenaventura, $M^{\mathrm{a}}$ \\ Neil A. Christie, MD ${ }^{a}$ \\ Chandra Belani, $\mathrm{MD}^{\mathrm{C}}$ \\ James D. Luketich, $\mathrm{MD}^{\mathrm{a}}$
}

From the Department of Surgery, Division of Thoracic and Foregut Surgery, University of Pittsburgh Medical Center, ${ }^{a}$ the Department of Biostatistics, University Pittsburgh Cancer Institute, ${ }^{\mathrm{b}}$ and the Division of Hematology and Oncology, University of Pittsburgh Medical Center and University of Pittsburgh Cancer Institute, ${ }^{\mathrm{C}}$ Pittsburgh, Pa.

Supported by a research grant from Boston Scientific Corporation, Boston, Mass.

Read at the Thirtieth Annual Meeting of The Western Thoracic Surgical Association, Maui, Hawaii, June 23-26, 2004.

Received for publication June 18, 2004; revisions received Sept 21, 2003; accepted for publication Oct 8, 2004.

Address for reprints: James D. Luketich, MD, Professor of Surgery, Chief, Division of Thoracic and Foregut Surgery, UPMC Presbyterian, 200 Lothrop St, Suite C-800, Pittsburgh, PA 15213 (E-mail: luketichjd@ upmc.edu).

J Thorac Cardiovasc Surg 2005;129:639-44 $0022-5223 / \$ 30.00$

Copyright $\odot 2005$ by The American Association for Thoracic Surgery

doi:10.1016/j.jtcvs.2004.10.019
Objective: Treatment options for patients with non-small cell lung cancer who are not surgical candidates or who refuse operation are limited. Radiofrequency ablation represents a potential less invasive option for these patients. Our initial experience with radiofrequency ablation for peripheral, primary non-small cell lung cancer is reported.

Methods: We treated 21 tumors in 18 patients. Median age was 75 (range 58-86) years. Cancer stages were I $(n=9)$, II $(n=2)$, III $(n=3)$, and IV $(n=4)$. Patients with stage IV disease included 3 with recurrence after previous lobectomies and 1 with a synchronous liver metastasis also treated with radiofrequency ablation. Median tumor diameter was $2.8 \mathrm{~cm}$ (range 1.2-4.5 cm). Radiofrequency ablation was delivered by minithoracotomy in 2 cases and by a computed tomographyguided percutaneous approach in 16 patients. Computed tomographic and positron emission tomographic scans were used to evaluate recurrence and radiographic response in ablated nodules.

Results: One postoperative death occurred from pneumonia after open radiofrequency ablation. Median hospital stay was 2.5 days. A chest tube or pigtail catheter was required in 7 patients $(38.9 \%)$ for procedure-related pneumothoraces. At a median follow-up of 14 months, 15 patients $(83.3 \%)$ were alive. Local progression occurred in 8 nodules $(38.1 \%)$. Mean and median progression-free intervals were 16.8 and 18 months, respectively. For stage I cancers, mean progression-free interval was 17.6 months. Median progression-free interval was not reached.

Conclusion: This study demonstrates the feasibility of radiofrequency ablation for small, peripheral non-small cell lung cancer tumors. Local control is comparable to, if not better than, that provided by radiotherapy. Radiofrequency ablation should continue to be evaluated by thoracic surgeons as a noninvasive therapy for the high-risk patient with non-small cell lung cancer.

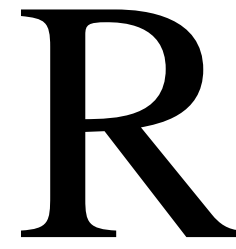

adiofrequency ablation (RFA) is a relatively new modality that has been successfully used for the treatment of hepatic tumors. ${ }^{1}$ During the past few years, an increasing number of reports have described the use of RFA for malignant lung nodules. ${ }^{2,3}$ Most of these have been case reports or small series describing primary efficacy and safety with minimal or no follow-up. We have previously described our early experience with RFA. ${ }^{4}$ In that initial series, most tumors were pulmonary 
TABLE 1. Modified RECIST criteria used to evaluate treatment response

\begin{tabular}{|c|c|c|c|}
\hline Response & CT mass size (RECIST) & CT mass quality & PET \\
\hline Complete (any 2) & $\begin{array}{l}\text { Lesion disappearance or scar } \\
<25 \% \text { original size }\end{array}$ & Cyst or cavity formation & SUV $<2.5$ \\
\hline & & Low density of entire lesion & \\
\hline Partial (any 1) & $\begin{array}{l}\text { Decrease of }>30 \% \text { in LD of } \\
\text { target lesion }\end{array}$ & $\begin{array}{l}\text { Central necrosis or central cavitation } \\
\text { with liquid density }\end{array}$ & $\begin{array}{l}\text { Decreased SUV or area of } \\
\text { FDG uptake }\end{array}$ \\
\hline Stable lesion (any 1) & $\begin{array}{l}\text { Decrease of }<30 \% \text { in LD of } \\
\text { target lesion }\end{array}$ & $\begin{array}{l}\text { Mass solid appearance, no central } \\
\text { necrosis or cavity }\end{array}$ & $\begin{array}{l}\text { Unchanged SUV or area of } \\
\text { FDG uptake }\end{array}$ \\
\hline Progression (any 2) & $\begin{array}{l}\text { Increase of }>20 \% \text { in LD of } \\
\text { target lesion }\end{array}$ & Solid mass, invasion adjacent structures & Higher SUV \\
\hline
\end{tabular}

Target lesions represent tumors treated with RFA. SUV, Standard uptake value of fluorodeoxyglucose F18 in PET scan; $L D$, largest diameter of target lesions; $F D G$, fluorodeoxyglucose F18.

\section{TABLE 2. Morbidity after RFA}

\begin{tabular}{llr}
\hline Complication & No. & $\%$ \\
\hline Pneumothorax & 7 & 38.9 \\
Pneumonia & $2^{*}$ & 11.1 \\
Air leak $>4$ d & 1 & 5.6 \\
Pulmonary embolus & 1 & 5.6
\end{tabular}

*Both pneumonias occurred after open RFA procedures.

metastases, with only a few cases of primary non-small cell lung cancer (NSCLC). The principal findings of our earlier report were that RFA was more effective for smaller $(\leq 5$ $\mathrm{cm})$ tumors, with better early survival and response to treatment. Additionally, in our previous report we described a modification of the Response Evaluation Criteria in Solid Tumors (RECIST) criteria (Table 1) that was used to assess treatment response and progression at the ablated sites. ${ }^{5,6}$ This article reports our ongoing experience with RFA for primary NSCLC and is part of an ongoing institutional review board-approved study that continues to accrue at the University of Pittsburgh. The aims of this study were to evaluate the efficacy of RFA in NSCLC and to determine progression-free survival at intermediate follow-up.

\section{Methods}

\section{Patient Selection}

A total of 18 patients with 21 NSCLC tumors underwent RFA. Patients included 10 men and 8 women. The median age was 75 years (range 58-86 years). Five of these patients were previously reported on in our initial pilot study, ${ }^{4}$ and 11 are part of an ongoing study. Inclusion criteria for this study included (1) target tumor 4 $\mathrm{cm}$ or smaller, (2) either evaluation by a thoracic surgeon that patient was at high risk for operation or patient refusal of operation, (3) stage I or II NSCLC, (4) recurrent NSCLC (same histologic type) after previous resection, and (5) persistent peripheral cancer after definitive chemotherapy or radiation therapy. Exclusion criteria included (1) central tumors defined as $3 \mathrm{~cm}$ or less from the hilum, (2) more than three tumors, and (3) malignant pleural effusion. Stages of disease included I $(n=9)$, II $(n=2)$,
III $(\mathrm{n}=3)$, and IV $(\mathrm{n}=4)$. The patients with stage IV disease included 3 with recurrent cancer after a previous lobectomy (tumor histologic type was the same as the original primary in all cases) and 1 patient with a synchronous liver metastasis that was also treated with RFA. Biopsy specimens were obtained in all cases either before or at the time of RFA. The exception was if there were any synchronous nodules present. If the computed tomographic CT and positron emission tomographic (PET) scans were consistent with a diagnosis of cancer, then only one nodule was sampled for biopsy. Tumor histologic types included squamous (n $=10)$, adenocarcinoma $(\mathrm{n}=5)$, adenosquamous $(\mathrm{n}=1)$, bronchiolar $(n=1)$, and large cell undifferentiated $(n=1)$. The reasons for performing RFA in preference to operation included poor pulmonary function $(\mathrm{n}=8)$, previous lung resection for cancer $(n=4)$, patient refusal of resection $(n=4)$, high cardiac risk $(n=3)$, and failure of definitive chemoradiation $(n=1)$. It should be noted that in some cases more than one factor influenced the decision to perform RFA. The median forced expiratory volume in 1 second, forced vital capacity, and diffusing capacity of carbon monoxide of the patients with impaired pulmonary function were $39.5 \%, 53 \%$, and $34 \%$, respectively. Of the 4 patients who had undergone previous resections ( 3 with stage IV and with a new primary NSCLC), 2 had impaired pulmonary function precluding any resection. The third patient had 2 metastases in the left lower lobe after a left upper lobectomy. After failure of chemotherapy, RFA was selected. The fourth patient had a left lower lobe recurrence develop after a previous left upper lobectomy and chest wall resection. Completion pneumonectomy was required, but because of concerns regarding the patient's ability to tolerate this, open RFA was performed. The 21 RFA procedures were performed during a 24-month period. During this time, approximately 760 lung resections for malignant tumors were performed by our group, representing about $2.7 \%$ of our current practice.

\section{RFA Technique}

All the RFA procedures were performed by thoracic surgeons either in the operating room or in the radiation oncology CT scan suite. The initial procedures were performed with an open approach through a minithoracotomy $(n=2)$. Subsequently, CTguided percutaneous ablation was performed in all other cases ( $\mathrm{n}$ $=16$ ). All procedures were performed with patients under general anesthesia. In the CT percutaneous cases, a finder needle (22- 


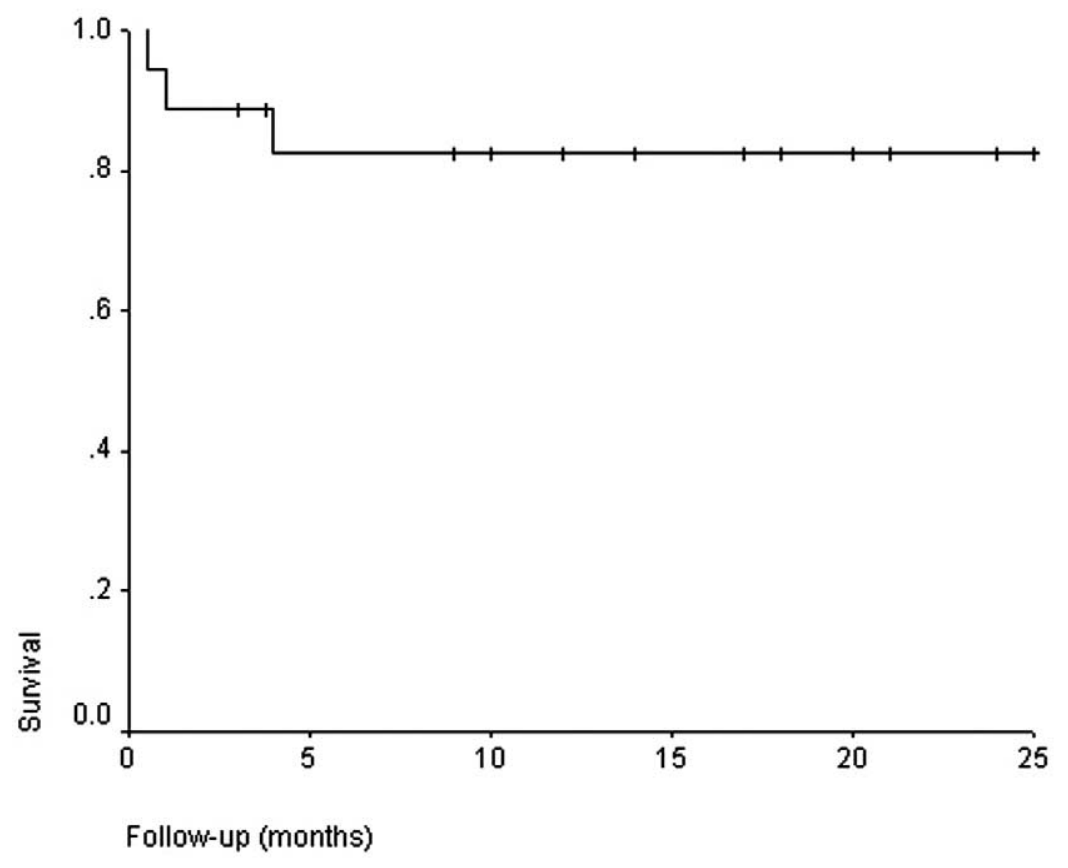

Figure 1. Overall survival after RFA for NSCLC $(n=18)$.

gauge long spinal needle) was used to determine the trajectory and placement of the active RFA probe. The LeVeen needle electrode (Boston Scientific Corporation, Boston, Mass) was selected according to the diameter of the target lesion and placed into the lesion parallel to the trajectory of the finder needle. Multiple tines were deployed to maximize the treatment area. Generally, we strove to achieve an ablation between 0.5 and $1 \mathrm{~cm}$ greater in diameter than the maximum diameter of the cancer. Therefore an active electrode at least $0.5 \mathrm{~cm}$ larger than the maximum diameter of the tumor was selected. In some cases, a coaxial needle was used to pass the active electrode (LeVeen electrode) into the lesion. The shorter length of the coaxial needle relative to the active electrode facilitated passage through the scanner, particularly for a very peripheral lesion. Additionally, in cases where a pretreatment tissue diagnosis was not available, it was possible to pass a true-cut needle through the coaxial needle sheath and obtain a biopsy specimen.

After RFA, CT scans and PET scans (in most patients) were performed at 4 to 6 weeks and then at 3-month intervals. The modified RECIST criteria described previously (Table 1) were used to assess response. Time to progression was classified as the time from ablation to the first sign of progressive disease. At least two surgeons reviewed the CT and PET scans in every case to determine whether progression had occurred.

\section{Statistical Analysis}

All data was entered into an SPSS file (version 11 for Windows; SPSS Inc, Chicago, Ill). The $\chi^{2}$ test was used for dichotomous variables. Kaplan-Meier with log-rank analysis was used to evaluate overall and progression-free survivals and to compare patients.

\section{Results}

A total of 21 tumors were treated in 18 patients. The median tumor diameter was $2.8 \mathrm{~cm}$ (range 1.2-4.5 cm). There was 1 perioperative death, that of a patient who had undergone open RFA of a right upper lobe lesion in conjunction with a right lower lobe lobectomy. This patient had a pulmonary embolus develop, and then pneumonia with subsequent sepsis and multisystem organ failure. Morbidity occurred in 10 patients $(55.6 \%)$ but was minor in most cases. Morbidity is detailed in Table 2. The most common complication was the need for a chest tube or pigtail catheter for pneumothorax in 7 cases (38.9\%). Usually the pneumothorax was minor in nature, with the tube removed on the first day after the RFA. For all patients, the median duration of chest tube placement was less than 24 hours (range 0-14 days). Median hospital stay was 2.5 days (1-35 days). The long chest-tube drainage of 14 days and hospital stay of 35 days occurred in 1 of the patients treated with open RFA. Median follow-up was 14 months (3-25 months) after RFA. Two deaths occurred among the 17 hospital survivors at 1 and 4 months. One death was related to chronic rejection after previous lung transplantation. This patient had stage I cancer; death occurred 4 months after RFA. The second death occurred in a patient with stage II cancer and chronic renal failure. Death occurred 1 month after RFA and was related to the underlying renal disease. The Kaplan-Meier survival curve for the 18 patients is demonstrated in Figure 1. Mean survival was 20.97 months; median survival was not 


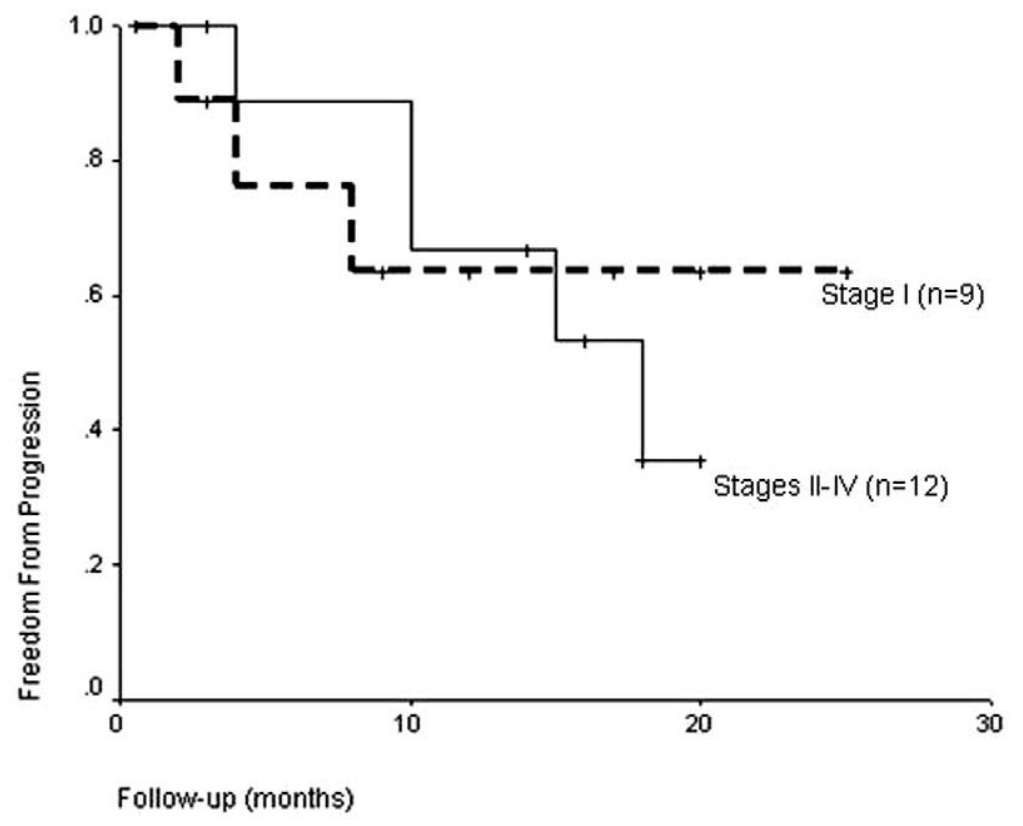

Figure 2. Progression-free interval in 21 lung nodules after RFA.

reached. Among the 21 lung nodules (in 18 patients), progression was seen in 8 nodules $(38.1 \%)$ in 6 (33\% patients). Salvage therapy in these 6 patients included chemotherapy and radiation $(n=2)$, repeated RFA $(n=1)$, chemotherapy and completion pneumonectomy $(\mathrm{n}=1)$, lobectomy $(\mathrm{n}=$ $1)$, and wedge resection $(n=1)$. The mean and median progression-free intervals were 16.8 and 18 months, respectively. Comparison of the stage I tumors with the higher stage cancers demonstrated a trend $(P=.885)$ favoring stage I (Figure 2). Mean progression-free interval was 17.6 months for stage I cancers. Median progression-free interval was not reached for stage I cancers. For the more advanced cancers, mean and median progression-free intervals were 14.98 and 18 months, respectively.

\section{Discussion}

RFA is a minimally invasive technique for treating pulmonary nodules that avoids the morbidity and mortality traditionally associated with pulmonary resection. ${ }^{7}$ Lobar resection should, however, continue to be the standard therapy for stage I NSCLC. Previous reports have demonstrated 5-year survivals as great as $80 \%^{8}$ and local recurrence rates of $5 \%$ after lobectomy for stage I NSCLC. ${ }^{9}$ For the moderately compromised patient, sublobar resection is an option. The main concern with sublobar resection is the increased local recurrence relative to lobectomy. ${ }^{9}$ Recent reports, however, suggest that the use of intraoperative adjuvant brachytherapy in combination with sublobar resection may reduce the higher local recurrence rate associated with sublobar resection alone. ${ }^{10,11}$
For those patients who are unable to tolerate pulmonary resection, external beam radiation has traditionally been used. Treatment results are inferior to those of resection. In a previous study of 71 node-negative patients who received at least 60 Gy to their cancers, ${ }^{12} 3$ and 5-year survivals were $19 \%$ and $12 \%$. Disease-free survivals were $44 \%$ and $32 \%$. A more recent report described results after radiation therapy in 60 patients with stage I or II cancers. ${ }^{13}$ Local progression occurred in $53 \%$ of patients, with a median progression-free survival of 18.5 months and an overall median survival of 20 months. RFA compares well with these results. In our series, when looking at all cancer stages combined, median progression-free interval was 18 months. For the stage I patients, median progression-free interval was not reached, and mean progression-free interval was 17.6 months.

Some of the difficulties with standard methods of radiation include the problems of radiation injury to surrounding tissues and the difficulties of delivering a precise area of radiation to a target area because of lung movement with respiration. Radiation pneumonitis is a significant and potentially life-threatening complication of radiation, particularly for an impaired patient who is unable to undergo resection. In one series of patients treated with definitive radiotherapy, this complication occurred in $8.3 \%$ of patients. ${ }^{13}$ RFA avoids some of these issues and additionally allows treatment to be given in a single setting. On the other hand, not all tumors are amenable to RFA. We previously reported a case of fatal hemoptysis occurring 21 days after RFA of a central tumor metastasis. ${ }^{4}$ Because of this, we do not recommend CT-guided RFA of central lesions close to 
the hilum. Additionally, some lesions may not be accessible to percutaneous approaches because of overlying structures, preventing an adequate window to the lesion.

Stereotactic radiosurgery is a relatively new approach that minimizes some of the problems with standard radiation therapy and allows delivery of higher doses of radiation to the targeted tumor. ${ }^{14}$ The accuracy is achieved by extremely precise spatial localization of the tumor and delivery of multiple cross-fired beams of directed radiation to converge upon the tumor. A recent study demonstrated a partial response in $87 \%$ and a complete response in $27 \%$ of 37 high-risk patients with stage I NSCLC after stereotactic radiosurgery. ${ }^{15}$ At a median follow-up of 15.2 months, there was local recurrence in 6 patients in this study $(16.2 \%){ }^{15}$ As RFA and stereotactic radiosurgery for NSCLC become more prevalent, further studies will be required to compare and determine the appropriate use of each modality. Because there is overlap in the types of tumors that are suitable for these nonresectional therapies, further studies will be needed to address issues such as long-term effectiveness, quality of life, and cost-effectiveness.

A problem that is challenging after RFA is the assessment of treatment response. Unlike an operation in which a cancer is removed, a residual mass associated with some degree of scarring is usual after RFA. In some cases, this scarring may involve a larger area than the original tumor. PET scans may continue to have positive results, making it difficult to assess whether viable tumor or scarring is present. We have been using a modification of the RECIST criteria to assess tumor progression at follow-up. ${ }^{4}$ Other centers have been using CT densitometry protocols to help evaluate persistent or recurrent disease. ${ }^{16}$ However, densitometric techniques are time-consuming and of value only for those patients with single nodules that have been ablated. Because the inflammatory changes after RFA have often started to subside by 3 months, the 3-month scan is often more useful as the baseline scan against which local progression can be judged. The American College of Surgeons Oncology Group will be using this same approach to assess local control after RFA in a study (Z4033) that is currently being developed. A goal of this study will be to establish a common approach to treatment and follow-up of ablated nodules in a multicenter setting.

\section{Conclusions}

RFA is a minimally invasive approach that can be used to treat NSCLC in patients who are not candidates for pulmonary resection. Intermediate results are encouraging and appear to be at least comparable to those of external beam radiation. As expected, treatment results are superior for stage I tumors. It is imperative that thoracic surgeons continue to evaluate ablative techniques such as RFA as these are introduced to clinical practice.

\section{References}

1. Curley SA, Izzo F, Delrio P, Ellis LM, Granchi J, Vallone $\mathrm{P}$, et al. Radiofrequency ablation of unresectable primary and metastatic hepatic malignancies: results in 123 patients. Ann Surg. 1999;230:1-8.

2. Dupuy DE, Zagoria RJ, Akerley W, Mayo-Smith WW, Kavanagh PV, Safran H. Percutaneous radiofrequency ablation of malignancies in the lung. AJR Am J Roentgenol. 2000;174:57-9.

3. Schaefer O, Lohrman C, Langer M. CT-guided radiofrequency ablation of a bronchogenic carcinoma. Br J Radiol. 2003;76:268-70.

4. Herrera LJ, Fernando HC, Perry Y, Gooding WE, Buenaventura PO, Christie NA, et al. Radiofrequency ablation of pulmonary malignant tumors in nonsurgical candidates. J Thorac Cardiovasc Surg. 2003; 125:929-37.

5. Padhani AR, Ollivier L. The RECIST (Response Evaluation Criteria in Solid Tumors) criteria: implications for diagnostic radiologists. $\mathrm{Br}$ Radiol. 2001;74:983-6.

6. Tsuchida Y, Therasse P. Response evaluation criteria in solid tumors (RECIST): new guidelines. Med Pediatr Oncol. 2001;37:1-3.

7. Ginsberg RJ, Hill LD, Eagan RT, Thomas P, Mountain CF, Deslauriers J, et al. Modern thirty-day operative mortality for surgical resections in lung cancer. J Thorac Cardiovasc Surg. 1983;86:654-8.

8. Thomas PA Jr, Rubinstein. Cancer recurrence after resection: T1 N0 non-small-cell lung cancer. Lung Cancer Study Group. Ann Thorac Surg. 1990;49:242-7.

9. Ginsberg RJ, Rubinstein LV. Randomized trial of lobectomy versus limited resection for T1 N0 non-small cell lung cancer. Lung Cancer Study Group. Ann Thorac Surg. 1995;60:615-22.

10. Santos R, Colonias A, Parda D, Trombetta M, Maley RH, Machery R, et al. Comparison between sublobar resection and 125I brachytherapy following sublobar resection in high-risk patients with stage I nonsmall cell cancer. Surgery. 2003;134:691-7.

11. Lee W, Daly BD, Di Petrillo TA, Morelli DM, Neuschatz AL, Morr J, et al. Limited resection for non-small cell lung cancer: observed local control with implantation of 125I brachytherapy seeds. Ann Thorac Surg. 2003;75:237-42.

12. Kupelian PA, Komaki R, Allen P. Prognostic factors in the treatment of node-negative non-small cell lung carcinoma with radiotherapy alone. Int J Radiat Oncol Biol Phys. 1996;36:607-13.

13. Zierhut D, Bettscheider C, Schubert K, van Kampen M, Wannenmacher M. Radiation therapy of stage I and II non-small cell lung cancer (NSCLC). Lung Cancer. 2001;34 Suppl 3:S39-43.

14. Whyte RI, Crownover R, Murphy MJ, Martin DP, Rice TW, DeCamp $\mathrm{MM} \mathrm{Jr}$, et al. Stereotactic radiosurgery for lung tumors: preliminary report of a phase I trial. Ann Thorac Surg. 2003;75:1097-101.

15. Timmerman R, Papiez L, McGarry R, Likes L, DesRosiers C, Frost S, et al. Extracranial stereotactic radioablation: results of a phase I study in medically inoperable stage I non-small cell lung cancer. Chest. 2003;124:1946-55.

16. Suh RD, Wallace AB, Sheehan RE, Heinze SB, Goldin JG. Unresectable pulmonary malignancies: CT-guided percutaneous radiofrequency ablation-preliminary results. Radiology. 2003;229:821-9.

\section{Discussion}

Dr John D. Mitchell (Denver, Colo). We have all become accustomed to new and innovative therapies coming from the thoracic group at the University of Pittsburgh, and your work with RFA is no exception. I am going to move right to my questions. First, you are using a 3-dimensional device to encompass a 3-dimensional object, the tumor, with in most of these cases a 2-dimensional imaging tool. How do you make sure that your treatment area completely covers or encompasses an often very spiculated lesion?

Dr Fernando. I think that is a very good question. There really is no other way of doing this. You have to use 2-dimensional imaging. What we do is use a probe that is slightly bigger than the lesion that we are treating, so for instance if we are treating a $2-\mathrm{cm}$ lesion, then we would use a $3-\mathrm{cm}$ diameter probe or $3.5-\mathrm{cm}$ diameter probe. With the CT scanner that we use, we do get 
images in a couple of different planes, not the planes that you are typically used to seeing on an image that you get generated in your office. I think this may be part of the reason why we do see local progression; we don't get quite a perfect ablation in all cases.

Dr Mitchell. Second, although the pneumothorax rate approached about $40 \%$, most were very minor in nature, and you are deploying a pretty good size needle and then deploying multiple tines out into what is often very emphysematous lung. Why don't you have more of a problem with persistent air leak in these patients?

Dr Fernando. First, I would bring to your attention that only about $50 \%$ of these patients had a pneumothorax. I think this was because some of these patients had previous surgery, so there were probably adhesions that prevented pneumothoraces. Also some patients had been exposed to asbestos, which may also have been associated with adhesions. I am surprised that pneumothoraces aren't a big problem. I have had 2 patients with severe emphysema who had never been operated on before in whom at the time of the procedure I put in a pigtail. The pigtail wasn't enough to control the pneumothorax. I put in one chest tube, and then actually two chest tubes, and in 1 case we actually had to make a blow hole postoperatively because the patient swelled up like a balloon. So we do see this problem, but not as much as you would expect. As I mentioned, with most of these patients we put a pigtail in at the time of the procedure and pulled it out the next day, and that was that.

Dr Mitchell. Third, you included a number of patients with stage IV disease in your series. Could you elaborate a little bit more on how you see RFA being used for stage IV disease?

Dr Fernando. I think this has to be in conjunction with your medical oncologists and radiation oncologists. Among our patients with stage IV disease, as I mentioned, there were 3 patients who had previously undergone lobectomy and had a new recurrence in another lobe with the same histologic type. These patients we decided to treat with RFA primarily. The fourth patient again was considered to have a low performance status, and the oncologist was reluctant to treat that patient with chemotherapy, so the patient underwent RFA ablation with laparoscopy for the liver lesion and then we performed the CT-guided ablation of the lung lesion. I think we are going to see more of these patients around. You know that as thoracic surgeons we don't typically operate on patients with stage IV disease, but as we start becoming familiar with these techniques and these techniques become more widespread, we are going to see more of these patients.

Dr Mitchell. Finally, it would seem that the major therapeutic alternative to RFA in medically inoperable patients with stage I disease is stereotactic radiotherapy, for which better and better survival results are being reported all the time. When and why would you advocate the use of RFA instead of stereotactic radiotherapy in these patients?

Dr Fernando. I don't think that the answer as which modality, sterotactic radiosurgery or RFA, is known yet. The same type of data that we are starting to see reported with RFA needs to be devoloped for sterotactic radiosurgery. In fact, we are actually working in conjunction with the thoracic group from Stanford on a protocol for sterotactic radiosurgery to try and look at long-term outcomes such as survival and time to progression. I think there is going to be a point in the future at which we will have to compare sterotactic radiosurgery with RFA. Some situations where we can't use RFA include very central nodules, because we worry about burning the tumor close to the hilum, and also very small tumors hidden behind bony structures, particularly at the apex of the lung. It is very hard to get a small tumor in the apex because of vascular structures as well as the first rib and clavicle hindering access. With sterotactic radiosurgery, you don't have to place a probe directly into the lesion itself. Instead, fiducials are placed around the tumor. The radiation oncology technician then uses these fiducials to map out the area of lung to be treated with sterotactic radiosurgery. 